\title{
Some Properties of Interval Quadratic Programming Problem
}

\author{
Qianqian $\mathrm{Xu}^{1}$, Shengnan $\mathrm{Jia}^{1}$, Haohao $\mathrm{Li}^{2,}{ }^{*}$, Jinhua Huang ${ }^{3}$ \\ ${ }^{1}$ School of Sciences, Hangzhou Dianzi University, Hangzhou, China \\ ${ }^{2}$ School of Data Sciences, Zhejiang University of Finance and Economics, Hangzhou, China \\ ${ }^{3}$ School of Automation Science and Engineering, South China University of Technology, Guangzhou, China
}

Email address:

hlzufe@126.com (Haohao Li)

${ }^{*}$ Corresponding author

\section{To cite this article:}

Qianqian Xu, Shengnan Jia, Haohao Li, Jinhua Huang. Some Properties of Interval Quadratic Programming Problem. International Journal of Systems Science and Applied Mathematics. Vol. 2, No. 5, 2017, pp. 105-109. doi: 10.11648/j.ijssam.20170205.15

Received: June 5, 2017; Accepted: June 27, 2017; Published: October 24, 2017

\begin{abstract}
For interval liner programming problems, Rohn proposed four equivalence relations regarding to the upper and lower bounds of the interval optimal value. In this paper, similar problems of interval quadratic programming problem have been discussed. Some interesting properties have been proved and an illustrative example and remarks are given to get an insight of the properties.
\end{abstract}

Keywords: Interval Quadratic Programming, Lower and Upper Bounds, Optimal Value

\section{Introduction}

Interval systems and interval optimal models are often used for modeling information systems and engineering problems, e.g. [1]. Over the past decades, the interval systems and interval mathematical programming (IvMP) have been studied by many authors, see e.g. [2-6, 20-27, 32-35] and the references therein. Some papers studied the problem of computing the range of optimal values of interval linear programming problems, see e.g., [12-17] among others. Some authors studied the problem of computing the range of optimal values of interval quadratic programs (IvQP) [7, 8, 18-19].

The other frequent problems is study the properties on upper and lower bounds of IvMP. There have been developed diverse methods for computing the lower and upper bounds of IvQP. Liu [18] and Li [19] described some methods to compute the lower and upper bounds of IvQP with inequality and nonnegative constraints. Hladik [7] focused on convex quadratic programming problems with interval data, the problem of computing the best case and the worst case optimal values was discussed for interval convex quadratic programming problems of certain forms, then he studied the method of the upper and lower bounds of interval-valued convex quadratic programming problems in a general form.
For computing the upper bound, these methods described in $[8,18,19]$ are based on the dual problem of IvQP (dual method for short), under the condition that the zero duality gap of a pair of primal and dual IvQP is specified. Recently, Li et al [11] proposed a new method to compute the upper bound of optimal values of IvQP, in this new method, only primal program is taken into consideration, the dual problem is not required and thus the condition that the duality gap is zero is also removed, and then $\mathrm{Li}$ described the properties on the upper and lower bounds of interval quadratic programming [9-11]. However few was done on the relations among the upper and lower bounds. The relations among the upper and lower bounds of IvLP (interval linear programming) have been established in $[4,8]$. In this paper, IvQP and several equivalent conditions for interval quadratic programming problem have been studied. First, some properties of interval quadratic program are formulated. Based on these results, some interesting and useful relations of interval quadratic program will be given, which give an insight into the corresponding problems.

\section{Preliminaries}

From notations from [4], an interval matrix is defined as 


$$
\boldsymbol{A}=[\underline{A}, \bar{A}]=\left\{A \in R^{m \times n} \mid \underline{A} \leq A \leq \bar{A}\right\}
$$

where $\underline{A}, \bar{A} \in R^{m \times n}, \quad \underline{A} \leq \bar{A}$, and $" \leq " \quad$ is understood componentwise. The center and the radius of matrix $\boldsymbol{A}$ is denoted by

$$
A_{c}=\frac{1}{2}(\underline{A}+\bar{A}), A_{\Delta}=\frac{1}{2}(\bar{A}-\underline{A})
$$

So $\boldsymbol{A}=\left[A_{c}-A_{\Delta}, A_{c}+A_{\Delta}\right] \quad$ [4]. An interval vector $\boldsymbol{b}=[\underline{b}, \bar{b}]=\left\{b \in R^{m} \mid \underline{b} \leq b \leq \bar{b}\right\}$ is understood as one-column interval matrix.

Let $\{ \pm 1\}^{m}$ be the set of all $\{-1,1\}$ m-dimensional vectors, i.e.

$$
\{ \pm 1\}^{m}=\left\{y \in R^{m}|| y \mid=e\right\}
$$

where $e=(1, \ldots, 1)^{T}$ is the m-dimensional vector of all 1's.

For a given $y \in\{ \pm 1\}^{m}$, let

$$
T_{y}=\operatorname{diag}\left(y_{1}, y_{2}, \ldots, y_{m}\right)
$$

denote the corresponding diagonal matrix. For each $x \in R^{n}$, its sign vector $\operatorname{sgn} x$ is defined by

$$
\begin{aligned}
(\operatorname{sgn} x)_{i}= & \left\{\begin{array}{cl}
1 & \text { if } x_{i} \geq 0 \\
-1 & \text { if } x_{i}<0
\end{array}\right. \\
& g(A, B, b, c, d, Q)=\sup \left\{-\frac{1}{2} u^{T} Q u-b^{T} v-d^{T} w \mid Q u+A^{T} v+B^{T} w+c \geq 0, v \geq 0\right\} .
\end{aligned}
$$

where $i=1,2, \cdots, n$. then $|x|=T_{z} x$, where $z=\operatorname{sgn} x \in\{ \pm 1\}^{n}$.

Given an interval matrix $\boldsymbol{A}=\left[A_{c}-A_{\Delta}, A_{c}+A_{\Delta}\right]$, for each $y \in\{ \pm 1\}^{m}$ and $z \in\{ \pm 1\}^{n}$, the matrices $A_{y z}=A_{c}-T_{y} A_{\Delta} T_{z}$ are defined.

Similarly for an interval vector $b=\left[b_{c}-b_{\Delta}, b_{c}+b_{\Delta}\right]$ and for each $y \in\{ \pm 1\}^{m}$, the vectors $b_{y}=b_{c}+T_{y} b_{\Delta}$ are defined.

Let $\quad A \in R^{m \times n}, B \in R^{k \times n}, b \in R^{m}, c \in R^{n}, d \in R^{k} \quad$ and $Q \in R^{n \times n}$, consider the quadratic programming problem $\min \frac{1}{2} x^{T} Q x+c^{T} x$ subject to $A x \leq b, B x=d, x \geq 0$,

where $Q$ is positive semidefinite. Briefly, the problem is rewritten as

$$
\min \left\{\frac{1}{2} x^{T} Q x+c^{T} x \mid A x \leq b, B x=d, x \geq 0\right\} .
$$

The Dorn dual problem[10] of the quadratic (1) is

$\max \left\{-\frac{1}{2} u^{T} Q u-b^{T} v-d^{T} w \mid Q u+A^{T} v+B^{T} w+c \geq 0, v \geq 0\right\}$

Let

$f(A, B, b, c, d, Q)=\inf \left\{\frac{1}{2} x^{T} Q x+c^{T} x \mid A x \leq b, B x=d, x \geq 0\right\}$,

The following lemmas will be used in the proof of the main results.

Lemma 1.1. [11]

$$
\underline{f}=\min \left\{\frac{1}{2} x^{T} \underline{Q} x+\underline{c}^{T} x \mid \underline{A} x \leq \bar{b}, \underline{B} x \leq \bar{d}, \bar{B} x \geq \underline{d}, x \geq 0\right\}
$$

and

$$
\bar{f}(\boldsymbol{A}, \boldsymbol{B}, \boldsymbol{b}, \boldsymbol{c}, \boldsymbol{d}, \boldsymbol{Q})=\sup _{y \in\{ \pm 1\}^{k}} f\left(\bar{A}, B_{y e}, \underline{b}, \bar{c}, d_{y}, \bar{Q}\right)
$$

Lemma 1.2. [9] Let $f(\boldsymbol{A}, \boldsymbol{B}, \boldsymbol{b}, \boldsymbol{c}, \boldsymbol{d}, \boldsymbol{Q})=-\infty$, then there exists a $B_{0} \in \boldsymbol{B}$, such that

$$
f\left(\underline{A}, B_{0}, \bar{b}, d, \underline{Q}\right) \in\{-\infty, \infty\}
$$

holds for each $d \in \boldsymbol{d}$.

Lemma 1.3. [10] It is hold that

$\bar{f}=\sup \{f(A, B, b, c, d, Q) \mid A \in \boldsymbol{A}, B \in \boldsymbol{B}, b \in \boldsymbol{b}, c \in \boldsymbol{c}, d \in \boldsymbol{d}, Q \in \boldsymbol{Q}\}$.

The upper bound of the optimal values of (2) is defined as $\bar{\varphi}=\sup \{g(A, B, b, c, d, Q) \mid A \in \boldsymbol{A}, B \in \boldsymbol{B}, b \in \boldsymbol{b}, c \in \boldsymbol{c}, d \in \boldsymbol{d}, Q \in \boldsymbol{Q}\}$. 


$$
\bar{\varphi}=\left\{\begin{array}{lll}
-\infty, & \text { if } \bar{f}=-\infty ; & (a) \\
\bar{f}, & \text { if } \bar{f} \text { is finite; } & (b) \\
\text { cannot dermine, } & \text { if } \bar{f}=\infty . & (\text { c })
\end{array}\right.
$$

Lemma 1.4. [9] Let $\underline{f}$ be finite and let $x^{*}$ be an optimal solution of the problem (6). then

$$
\underline{f}=f\left(\underline{A}, B_{c}-T_{y} B_{\Delta}, \bar{b}, \underline{c}, d_{c}+T_{y} d_{\Delta}, \underline{Q}\right),
$$

where, for arbitrary $\alpha \in[-1,1]$

$$
y_{i}=\left\{\begin{array}{ll}
\frac{\left(B_{c} x^{*}-d_{c}\right)_{i}}{\left(B_{\Delta} x^{*}+d_{\Delta}\right)_{i}} & \text { if }\left(B_{\Delta} x^{*}+d_{\Delta}\right)_{i}>0 \\
\alpha & \text { if }\left(B_{\Delta} x^{*}+d_{\Delta}\right)_{i}=0
\end{array} \quad(i=1,2, \ldots, k)\right.
$$

\section{Some Properties of IvQP}

For an interval linear programming problem (IvLP) with data $\boldsymbol{A}, \boldsymbol{b}, \boldsymbol{c}$, Rohn proved that the following assertions are equivalent [4].

a. For each $A \in \boldsymbol{A}, B \in \boldsymbol{B}, b \in \boldsymbol{b}, c \in \boldsymbol{c}$ the problem

$$
\min \left\{c^{T} x \mid A x=b, x \geq 0\right\}
$$

has an optimal solution.

b. Both $\underline{f}(\boldsymbol{A}, \boldsymbol{b}, \boldsymbol{c})$ and $\bar{f}(\boldsymbol{A}, \boldsymbol{b}, \boldsymbol{c})$ are finite.

c. Both $\underline{f}(\boldsymbol{A}, \boldsymbol{b}, \boldsymbol{c})$ and $\bar{\varphi}(\boldsymbol{A}, \boldsymbol{b}, \boldsymbol{c})$ are finite.

d. The system

$$
\bar{A}^{T} p_{1}-\underline{A}^{T} p_{2} \leq \underline{c}
$$

is feasible and $\bar{\varphi}(\boldsymbol{A}, \boldsymbol{b}, \boldsymbol{c})$ is finite.

Similarly, the relationship of the following assertions is studied.

(a) For each $A \in \boldsymbol{A}, B \in \boldsymbol{B}, b \in \boldsymbol{b}, c \in \boldsymbol{c}, d \in \boldsymbol{d}, Q \in \boldsymbol{Q}$ the problem

$$
\min \left\{\frac{1}{2} x^{T} Q x+c^{T} x \mid A x \leq b, B x=d, x \geq 0\right\}
$$

has an optimal solution.

(b) Both $\underline{f}(\boldsymbol{A}, \boldsymbol{B}, \boldsymbol{b}, \boldsymbol{c}, \boldsymbol{d}, \boldsymbol{Q})$ and $\bar{f}(\boldsymbol{A}, \boldsymbol{B}, \boldsymbol{b}, \boldsymbol{c}, \boldsymbol{d}, \boldsymbol{Q})$ are finite.

(c) Both $\underline{f}(\boldsymbol{A}, \boldsymbol{B}, \boldsymbol{b}, \boldsymbol{c}, \boldsymbol{d}, \boldsymbol{Q})$ and $\bar{\varphi}(\boldsymbol{A}, \boldsymbol{B}, \boldsymbol{b}, \boldsymbol{c}, \boldsymbol{d}, \boldsymbol{Q})$ are finite.

(d) The system

$$
\underline{Q u}+\underline{A}^{T} v_{1}+\underline{B}^{T} v_{2}-\bar{B}^{T} v_{3}+\underline{c} \geq 0, v_{i} \geq 0, i=1,2,3
$$

is solvable, and $\bar{\varphi}(\boldsymbol{A}, \boldsymbol{B}, \boldsymbol{b}, \boldsymbol{c}, \boldsymbol{d}, \boldsymbol{Q})$ is finite.

Theorem 2.1. For an interval quadratic programming problem with data $A \in \boldsymbol{A}, B \in \boldsymbol{B}, b \in \boldsymbol{b}, c \in \boldsymbol{c}, d \in \boldsymbol{d}, Q \in \boldsymbol{Q}$ there holds that

$$
(a) \Rightarrow(b) \Leftrightarrow(c) \Rightarrow(d)
$$

Proof. $(a) \Rightarrow(b)$ : Since each problem (4) has an optimal solution, it must be $f(\boldsymbol{A}, \boldsymbol{B}, \boldsymbol{b}, \boldsymbol{c}, \boldsymbol{d}, \boldsymbol{Q})<\infty$. From Lemma 1.2, if $\underline{f}(\boldsymbol{A}, \boldsymbol{B}, \boldsymbol{b}, \boldsymbol{c}, \boldsymbol{d}, \boldsymbol{Q})=-\infty$, then there exists a $B_{0} \in \boldsymbol{B}$, such that $f\left(\underline{A}, B_{0}, \bar{b}, d, \underline{Q}\right) \in\{-\infty, \infty\}$, hold for each $d \in \boldsymbol{d}$, so the possibility of $\underline{f}(\boldsymbol{A}, \boldsymbol{B}, \boldsymbol{b}, \boldsymbol{c}, \boldsymbol{d}, \boldsymbol{Q})=-\infty$ is precluded by Lemma 1.2 . Hence $f(\boldsymbol{A}, \boldsymbol{B}, \boldsymbol{b}, \boldsymbol{c}, \boldsymbol{d}, \boldsymbol{Q})$ is finite. From Lemma 1.4 and Lemma 1.1, which can be got is that $\quad \underline{f}=f\left(\underline{A}, B_{c}-T_{y} B_{\Delta}, \bar{b}, \underline{c}, d_{c}+T_{y} d_{\Delta}, \underline{Q}\right) \quad$ and $\bar{f}=\sup _{y \in\{ \pm 1\}^{k}} \bar{f}\left(\bar{A}, B_{y e}, \underline{b}, \bar{c}, d_{y}, \bar{Q}\right)$, in other words, $\bar{f}$ gets the maximum in a finite group generated by $y$, hence $\bar{f}(A, B, b, c, d, Q)$ is finite.

$(b) \Rightarrow(c)$ : From Lemma 1.3 , if $\bar{f}(\boldsymbol{A}, \boldsymbol{B}, \boldsymbol{b}, \boldsymbol{c}, \boldsymbol{d}, \boldsymbol{Q})$ is finite, $\quad \bar{f}(\boldsymbol{A}, \boldsymbol{B}, \boldsymbol{b}, \boldsymbol{c}, \boldsymbol{d}, \boldsymbol{Q})=\bar{\varphi}(\boldsymbol{A}, \boldsymbol{B}, \boldsymbol{b}, \boldsymbol{c}, \boldsymbol{d}, \boldsymbol{Q}) \quad$. Hence $\bar{f}(\boldsymbol{A}, \boldsymbol{B}, \boldsymbol{b}, \boldsymbol{c}, \boldsymbol{d}, \boldsymbol{Q})$ is finite implies that $\bar{\varphi}(\boldsymbol{A}, \boldsymbol{B}, \boldsymbol{b}, \boldsymbol{c}, \boldsymbol{d}, \boldsymbol{Q})$ is finite.

According to the process of above proof, something can be easily got that if $f(\boldsymbol{A}, \boldsymbol{B}, \boldsymbol{b}, \boldsymbol{c}, \boldsymbol{d}, \boldsymbol{Q})$ is finite, then $\bar{f}(\boldsymbol{A}, \boldsymbol{B}, \boldsymbol{b}, \boldsymbol{c}, \boldsymbol{d}, \boldsymbol{Q})$ is finite. Thus, the result of $(c) \Rightarrow(b)$ is obviously true, since that if $\underline{f}(\boldsymbol{A}, \boldsymbol{B}, \boldsymbol{b}, \boldsymbol{c}, \boldsymbol{d}, \boldsymbol{Q})$ and $\bar{\varphi}(\boldsymbol{A}, \boldsymbol{B}, \boldsymbol{b}, \boldsymbol{c}, \boldsymbol{d}, \boldsymbol{Q}) \quad$ are $\quad$ finite, $\quad f(\boldsymbol{A}, \boldsymbol{B}, \boldsymbol{b}, \boldsymbol{c}, \boldsymbol{d}, \boldsymbol{Q}) \quad$ and $\bar{f}(\boldsymbol{A}, \boldsymbol{B}, \boldsymbol{b}, \boldsymbol{c}, \boldsymbol{d}, \boldsymbol{Q})$ are obviously finite.

Thus, (b) and (c) are necessary and sufficient conditions to each other.

$$
(c) \Rightarrow(d): \text { Since }
$$$$
\underline{f}=\inf \left\{\frac{1}{2} x^{T} \underline{Q} x+\underline{c}^{T} x \mid \underline{A} x \leq \bar{b}, \underline{B} x \leq \bar{d}, \bar{B} x \geq \underline{d}, x \geq 0\right\}
$$

The Dorn dual problem of (6) is

$$
\max \left\{-\frac{1}{2} u^{T} \underline{Q u}-\bar{b}^{T} v_{1}-\bar{d}^{T} v_{2}+\underline{d}^{T} v_{3} \mid \underline{Q u}+\underline{A}^{T} v_{1}+\underline{B}^{T} v_{2}-\bar{B}^{T} v_{3}+\underline{c} \geq 0, v_{i} \geq 0, i=1,2,3\right\}
$$

If $\underline{f}(A, B, b, c, d, Q)$ is finite, then by the strong duality theory [9], the following formula is established 


$$
\underline{f}=\sup \left\{-\frac{1}{2} u^{T} \underline{Q u}-\bar{b}^{T} v_{1}-\bar{d}^{T} v_{2}+\underline{d}^{T} v_{3} \mid \underline{Q u}+\underline{A}^{T} v_{1}+\underline{B}^{T} v_{2}-\bar{B}^{T} v_{3}+\underline{c} \geq 0, v_{i} \geq 0, i=1,2,3\right\}
$$

so that (7) is finite, thus the system (5) is solvable.

\section{An Illustrative Example}

In this section, an illustrative example is given for Theorem 2.1, which helps us to understand Theorem 2.1.

Example 1 Consider the interval quadratic program

$$
\begin{gathered}
\min \left[\frac{1}{2}, 1\right] x_{1}+x_{2} \\
\left\{\begin{array}{c}
{[1,2] x_{1}-x_{2}=[0,2]} \\
{[1,2] x_{1}-x_{2}=[-2,2]} \\
x_{1}, x_{2} \geq 0
\end{array}\right.
\end{gathered}
$$

The corresponding interval matrices and vectors of (9) are

$$
c=\left(\begin{array}{c}
\frac{1}{2} \\
1
\end{array}\right) \quad B=\left(\begin{array}{ll}
{[1,2]} & -1 \\
{[1,2]} & -1
\end{array}\right) \quad d=\left(\begin{array}{c}
{[0,2]} \\
{[-2,2]}
\end{array}\right)
$$

The lower bound of the optimal values can be determined by convex quadratic program

$$
\begin{gathered}
\min \frac{1}{2} x_{1}+x_{2} \\
\left\{\begin{array}{c}
2 x_{1}-x_{2} \geq 0 \\
x_{1}-x_{2} \leq 2 \\
2 x_{1}-x_{2} \geq-2 \\
x_{1}-x_{2} \leq 2 \\
x_{1}, x_{2} \geq 0
\end{array}\right.
\end{gathered}
$$

It can be shown that $\underline{f}=0$

Then the upper bound is computed by Lemma 1.1. This problem can be decomposed into four convex quadratic programs

$$
\begin{aligned}
& f_{1}=\min x_{1}+x_{2} \\
& \left\{\begin{array}{c}
2 x_{1}-x_{2}=0 \\
2 x_{1}-x_{2}=-2 \\
x_{1}, x_{2} \geq 0
\end{array}\right. \\
& f_{2}=\min x_{1}+x_{2} \\
& \left\{\begin{array}{c}
2 x_{1}-x_{2}=0 \\
x_{1}-x_{2}=2 \\
x_{1}, x_{2} \geq 0
\end{array}\right. \\
& f_{3}=\min x_{1}+x_{2}
\end{aligned}
$$

[2] R. E. Moore, R. B. Kearfott, M. J. Cloud, Introduction to interval analysis, SIAM, Philadelphia, 2009.

[3] A. Neumaier, Interval Methods for Systems of Equations, Cambridge University Press, Cambridge, 1990.

[4] Fiedler M, Rohn J, Nedoma J. Linear optimization problems with inexact date [M]. New York: Springer, 2006:35-66. 
[5] Rohn J. A general method for enclosing solutions of interval linear equations [J]. Optimization Letters, 2012, 6(4): 709-717.

[6] S. P. Shary, A new technique in systems analysis under interval uncertainty and ambiguity, Reliab. Comput. 8(5) (2002), pp. $321-418$.

[7] Hladík M. Interval convex quadratic programming problems in a general form [J]. Central European Journal of Operations Research, 2016: 1-13.

[8] Hladik M. Optimal value bounds in nonlinear programming with interval data[J]. Top, 2011, 19(1): 93-106.

[9] Li W, Jin J, Xia M, et al. Some properties of the lower bound of optimal values in interval convex quadratic programming $[\mathrm{J}]$. Optimization Letters, 1-16.

[10] Li W, Xia M, Li H. Some results on the upper bound of optimal values in interval convex quadratic programming [J]. Journal of Computational and Applied Mathematics, 2016, 302: 38-49.

[11] Li W, Xia M, Li H. New method for computing the upper bound of optimal value in interval quadratic program $[\mathrm{J}]$. Journal of Computational and Applied Mathematics, 2015,(288):70-80.

[12] Chinneck J W, Ramadan K. Linear programming with interval coefficients $[\mathrm{J}]$. Journal of the operational research society, 2000: 209-220.

[13] Hladik M. Interval linear programming: A survey [J]. Linear programming-new frontiers in theory and applications, 2012: 85-120.

[14] Mráz F. Calculating the exact bounds of optimal valuesin LP with interval coefficients [J]. Annals of Operations Research, 1998, 81: 51-62.

[15] Fiedler M, Nedoma J, Ramik J, et al. Linear optimization problems with inexact data $[\mathrm{M}]$. Springer Science \& Business Media, 2006.

[16] Hladík M. Optimal value range in interval linear programming [J]. Fuzzy Optimization and Decision Making, 2009, 8(3): 283-294.

[17] Hladík M. On approximation of the best case optimal value in interval linear programming [J]. Optimization Letters, 2014, 8(7): 1985-1997.

[18] Liu S T, Wang R T. A numerical solution method to interval quadratic programming $[\mathrm{J}]$. Applied mathematics and computation, 2007, 189(2): 1274-1281.

[19] Li W, Tian X. Numerical solution method for general interval quadratic programming $[\mathrm{J}]$. Applied mathematics and computation, 2008, 202(2): 589-595.

[20] Wu X Y, Huang G H, Liu L, et al. An interval nonlinear program for the planning of waste management systems with economies-of-scale effects - a case study for the region of
Hamilton, Ontario, Canada [J]. European Journal of Operational Research, 2006, 171(2): 349-372.

[21] Li W, Liu X, Li H. Generalized solutions to interval linear programmes and related necessary and sufficient optimality conditions [J]. Optimization Methods and Software, 2015, 30(3): 516-530.

[22] Prokopyev O A, Butenko S, Trapp A. Checking solvability of systems of interval linear equations and inequalities via mixed integer programming $[\mathrm{J}]$. European Journal of Operational Research, 2009, 199(1): 117-121.

[23] Inuiguchi M, Ramik J, Tanino T, et al. Satisficing solutions and duality in interval and fuzzy linear programming [J]. Fuzzy Sets and Systems, 2003, 135(1): 151-177.

[24] Li W, Luo J, Wang Q, et al. Checking weak optimality of the solution to linear programming with interval right-hand side [J]. Optimization Letters, 2014, 8(4): 1287-1299.

[25] Ishibuchi $H$, Tanaka $H$. Multiobjective programming in optimization of the interval objective function [J]. European journal of operational research, 1990, 48(2): 219-225.

[26] Shary S P. A new technique in systems analysis under interval uncertainty and ambiguity [J]. Reliable computing, 2002, 8(5): $321-418$.

[27] Neumaier A. Interval methods for systems of equations [M]. Cambridge university press, 1990.

[28] Steuer R E. Algorithms for linear programming problems with interval objective function coefficients [J]. Mathematics of Operations Research, 1981, 6(3): 333-348.

[29] Gabrel V, Murat C, Remli N. Linear programming with interval right hand sides [J]. International Transactions in Operational Research, 2010, 17(3): 397-408.

[30] Hladík M. How to determine basis stability in interval linear programming [J]. Optimization Letters, 2014, 8(1): 375-389.

[31] Allahdadi M, Nehi H M. The optimal solution set of the interval linear programming problems [J]. Optimization Letters, 2013, 7(8): 1893-1911.

[32] Hladík M. Weak and strong solvability of interval linear systems of equations and inequalities [J]. Linear Algebra and its Applications, 2013, 438(11): 4156-4165.

[33] Li W, Wang H, Wang Q. Localized solutions to interval linear equations $[\mathrm{J}]$. journal of computational and applied mathematics, 2013, 238: 29-38.

[34] Luo J, Li W. Strong optimal solutions of interval linear programming $[\mathrm{J}]$. Linear Algebra and its Applications, 2013, 439(8): 2479-2493.

[35] Wang X, Huang G. Violation analysis on two-step method for interval linear programming [J]. Information Sciences, 2014, 281: 85-96. 